\section{EL CÁNCER DE PULMÓN EN MUJERES}

\author{
Enriqueta Felip \\ Hospital Universitari Vall d'Hebron \\ efelip@vhebron.net
}

Cómo citar este artículo/Citation: Felip, E. (2015). “El cáncer de pulmón en mujeres". Arbor, 191 (773): a235. doi: http:// dx.doi.org/10.3989/arbor.2015.773n3005

Recibido: 9 diciembre 2014. Aceptado: 24 abril 2015.

RESUMEN: El cáncer de pulmón es la enfermedad maligna más frecuente en el mundo. En los últimos diez años en España, la mortalidad por cáncer de pulmón ha aumentado un $20 \%$ en las mujeres frente a un $5 \%$ en los hombres, debido a los cambios recientes de patrones de hábito tabáquico entre ambos sexos. El tabaco sigue siendo la causa única más importante de una enfermedad prevenible en un alto porcentaje de casos. Es muy importante que la población conozca esta realidad y que modifique el estilo de vida, eliminado el tabaquismo, para poder detener esta epidemia que representa el cáncer de pulmón. En este artículo se revisarán aspectos epidemiológicos, así como factores de riesgo, características y pronóstico de las mujeres con cáncer de pulmón, con una especial atención a la población española.

PALABRAS CLAVE: adenocarcinoma; EGFR; tabaco.

\section{LUNG CANCER AMONG WOMEN}

Copyright: (C) 2015 CSIC. Este es un artículo de acceso abierto distribuido bajo los términos de la licencia Creative Commons Attribution-Non Commercial (by-nc) Spain 3.0.

ABSTRACT: Lung cancer is the most frequent malignant disease in the world. Over the last ten years lung cancer mortality has risen in Spain by $20 \%$ among women compared with $5 \%$ among men. This is due to recent changes in both genders' smoking habits. Tobacco is still the single most important cause of a preventable disease in a high percentage of cases. It is extremely important that the population is aware of this and that people modify their lifestyle accordingly, giving up smoking in order to halt the lung cancer epidemic. In this article, we review epidemiological aspects, as well as risk factors, characteristics and the prognosis for women with lung cancer, focusing particularly on the Spanish population.

KEYWORDS: adenocarcinoma; EGFR; tobacco. 


\section{INTRODUCCIÓN}

El cáncer de pulmón representa en la actualidad la primera causa de muerte por cáncer. La incidencia del cáncer de pulmón a nivel mundial está disminuyendo entre los hombres pero está aumentado entre las mujeres debido a los cambios recientes de los patrones de hábito tabáquico entre ambos sexos. A pesar de los esfuerzos en prevención, el tabaco sigue siendo la causa única más importante de una enfermedad prevenible en un alto porcentaje de casos. En los últimos diez años en España, la mortalidad por cáncer de pulmón ha aumentado un $20 \%$ en las mujeres frente a un $5 \%$ en los hombres. A parte del tabaco, otros factores que se han analizado en relación a un posible mayor riesgo en desarrollar un cáncer de pulmón son aspectos de predisposición genética y exposición a factores hormonales aunque en la actualidad no hay datos concluyentes de ninguno de estos factores. En este capítulo se revisarán aspectos epidemiológicos y de evolución de las pacientes mujeres con cáncer de pulmón, con una especial atención a la población española.

\section{EPIDEMIOLOGÍA DEL CÁNCER DE PULMÓN EN MUJERES}

El cáncer de pulmón es la enfermedad maligna más frecuente en el mundo. En hombres, el cáncer de pulmón representa el tumor más frecuente (1,1 millones de casos, 16,5\% del total) (Ferlay et al., 2013; Malvezzi et al., 2014; Steliarova-Foucher, O'Callaghan y Ferlay, 2012; Torre et al., 2014). La tasas de incidencia en mujeres son generalmente más bajas que en hombres. Sin embargo, el cáncer de pulmón en las mujeres es el cuarto más frecuente en el mundo ( 513.000 casos, $8,5 \%$ de todos los cánceres) y representa la segunda causa de muerte por cáncer (427.000 muertes, 12,8\% de todos los cánceres). En Estados Unidos el cáncer de pulmón es ya la primera causa de muerte por cáncer entre mujeres por delante del cáncer de mama.

En España, el cáncer de pulmón constituye la tercera causa de muerte por cáncer en mujeres, solo por detrás del cáncer de mama y del cáncer colorrectal (Sánchez et al., 2010). En el año 2010, 3.452 mujeres en España fallecieron por cáncer de pulmón (8,4\% del total de las muertes por cáncer) y 17.303 hombres (26\% del total de las muertes por cáncer). La mortalidad en mujeres en nuestro país es más baja que en los países de nuestro entorno, aunque se observa una importante tendencia al alza. Según las últimas estimaciones, en nuestro país se diagnostican unos 25.500 nuevos cánceres de pulmón al año, 5.230 en mujeres.
En España se estima que la incidencia de cáncer de pulmón en mujeres aumenta un 4,4\% anual.

\section{FACTORES DE RIESGO DEL CÁNCER DE PULMÓN}

El tabaco es el principal factor de riesgo para desarrollar un cáncer de pulmón, estimándose que es el responsable del $80-90 \%$ de los casos de cáncer de pulmón en hombres y del $60-80 \%$ en mujeres (LortetTieulent et al., 2014; Ng et al., 2014; Papadopoulous, Guida y Leffondré, 2014). El riesgo de desarrollar un cáncer de pulmón es entre 15 y 30 veces mayor para una persona fumadora que para una no fumadora. El tabaquismo en la mujer es uno de los problemas sanitarios más importantes a nivel mundial (Martínez-Sánchez et al., 2010). Aunque la evolución del tabaquismo en cada sociedad sigue un patrón propio según las diferentes circunstancias, generalmente se ha visto que cuando el tabaco llega a una sociedad los primeros en iniciarse en su consumo son los varones, cuando el consumo de tabaco se ha extendido entre la población masculina es cuando las mujeres empiezan el consumo. En el último siglo ha habido algunas campañas agresivas de marketing de compañías tabacaleras cuyo objetivo son las mujeres y que se han acompañado de un incremento en las enfermedades relacionadas con el tabaco entre mujeres. Otro punto importante es la edad de inicio del hábito tabáquico. En España, la edad de inicio al consumo del tabaco se produce entre los 13 y los 14 años (Fernández et al., 2003). La prevención del inicio del tabaquismo debe ser una prioridad y las iniciativas en este sentido deben incluir la adolescencia. Entender la percepción de las mujeres hacia el tabaco puede ayudar a identificar maneras de reducir la prevalencia (Li et al., 2010).

El conocimiento sobre el riesgo de cáncer de pulmón asociado al tabaco se analizó en la población Española en una encuesta efectuada a 1.000 adultos Españoles en 2011. El 58\% de los participantes citaron el cáncer de pulmón como la primera enfermedad relacionada con el tabaco y 9 de cada 10 participantes conocían la relación entre el tabaco y el cáncer de pulmón (Majem et al., 2012)2012. La proporción de españoles de más de 15 años que fumaban cada día era todavía muy alta ( $27,9 \%$ en hombres, $20,2 \%$ en mujeres). Hay otros factores que se han relacionado con el riesgo a desarrollar cáncer de pulmón entre los que se incluye el contacto con el asbesto, arsénico, cromo, cadmio y níquel. Algunos factores ambientales como puede ser la exposición al radón también se han relacionado con un aumento de la frecuencia de riesgo a desarrollar cáncer de pulmón. 
Algunos estudios han sugerido que las mujeres podrían tener una mayor susceptibilidad para desarrollar cáncer de pulmón (Freedman et al., 2008; Henschke, Yip y Miettinen, 2006; Hsu et al. , 2009; Zang y Wynder, 1996). Estas diferencias se podrían explicar por tener una metabolización del tabaco diferente, aunque este es también un punto controvertido. Al comparar el cáncer de pulmón entre hombres y mujeres, el efecto de los estrógenos circulantes es la diferencia biológica clínicamente más relevante. La estimulación de estrógenos provoca la proliferación de líneas celulares de cáncer a través del receptor de estrógeno (Rouquette et al., 2012). Los receptores de estrógenos se pueden expresar tanto en el tejido normal del pulmón y en los tumores de pulmón en ambos sexos. Sin embargo, los patrones de expresión de los receptores hormonales según el sexo y la condición de fumador, o su factor pronóstico siguen siendo indefinidos. Globalmente los datos de los estudios publicados son controvertidos y los análisis prospectivos no apoyarían que las mujeres tuvieran una mayor susceptibilidad al cáncer de pulmón debido al tabaco.

También hay que tener en cuenta que el cáncer de pulmón en no fumadores es una entidad importante ya que representa a nivel mundial la séptima causa de muerte por cáncer cuando se considera de forma independiente (Mazieres et al., 2013b). A nivel europeo el cáncer de pulmón en no fumadores representa la 17 causa de mortalidad por cáncer, la novena causa de muerte por cáncer entre mujeres. Estudios epidemiológicos indican que la proporción de cánceres de pulmón en no fumadores es superior en mujeres que en hombres. En Europa el $21 \%$ de los cánceres de pulmón se diagnostican en mujeres no fumadoras comparado con el $2 \%$ de cánceres de pulmón en hombres no fumadores. A nivel molecular el cáncer de pulmón en no fumadores tiene unas alteraciones moleculares diferentes cuando se compara con el cáncer de pulmón de fumadores.

\section{CARACTERÍSTICAS DEL CÁNCER DE PULMÓN EN MUJERES}

En los últimos años hemos pasado de un predominio del carcinoma escamoso a la situación actual en el que subtipo más frecuente es el adenocarcinoma en ambos sexos (Khuder, 2001). La mayor parte de los estudios epidemiológicos ponen en evidencia que el adenocarcinoma de pulmón es el tipo histológico más frecuente en la mujer. En cuanto al resto de subtipos histológicos, el carcinoma de células pequeñas parece mostrar un aumento de incidencia solo entre las mujeres.
El riesgo de desarrollar cáncer de pulmón aumenta con la edad (Radzikowska, Glaz y Roszkowski, 2002). Sin embargo, las mujeres suelen ser diagnosticadas a edades más tempranas que los hombres y representan una mayor proporción de pacientes menores de 50 años. Estos datos han sido confirmados en España mediante el estudio epidemiológico prospectivo y multicéntrico "WORLD 07 base de datos de cáncer de pulmón-femenino en España" que incluye información de más de 2000 mujeres españolas diagnosticadas de cáncer de pulmón (Isla et al., 2012). De los resultados de este estudio destacamos que la edad media al diagnóstico fue de 61,9 años. Aproximadamente el $60 \%$ de las pacientes eran fumadoras. El tipo histológico más frecuente fue el adenocarcinoma (68\%) y más del $40 \%$ de las pacientes tenían una historia familiar de cáncer.

Las mutaciones de oncogenes relacionados con el cáncer de pulmón pueden tener diferentes prevalencias según el género (Dogan et al., 2012; Marchetti et al., 2011; Mazieres et al., 2013a; Paik et al., 2012). La mutación del gen del factor de crecimiento epidérmico (EGFR) está presente en aproximadamente el $16 \%$ de los pacientes de raza blanca con diagnóstico de adenocarcinoma de pulmón metastásico. Estas mutaciones son más frecuentes en mujeres y en no fumadores. En el cáncer de pulmón, casi el $90 \%$ de las mutaciones de EGFR son mutaciones L858R o deleciones en el exón 19. Sabemos que hasta un $60 \%$ de adenocarcinomas de mujeres no fumadoras presentan la mutación de EGFR. Se han encontrado de forma infrecuente mutaciones de Her2 con un gran predominio en mujeres. Otras mutaciones que podrían estar influenciadas por el género son las mutaciones de $B R A F$, translocaciones de EML4-ALK y translocaciones ROS1. Hay que tener en cuenta que el hecho de que algunas alteraciones moleculares sean más frecuentes en mujeres puede ser debido a una mayor proporción de cáncer de pulmón en mujeres no fumadoras que en hombres y no debido a una relación directa con el género.

\section{PRONÓSTICO DEL CÁNCER DE PULMÓN EN MUJERES}

Una serie de estudios indican que la supervivencia del cáncer de pulmón es mejor en mujeres que en hombres, independientemente del estadio, la histología, o el tratamiento (Fu et al., 2005; Janjigian et al., 2010; Micheli et al., 2009; Nakamura et al., 2011; Ries et al., 2007; Sagerup, Smastuen y Johannesen, 2001; Salmeron et al., 2012; Wakelee et al., 2012; WheatleyPrice et al., 2010). Según los datos de la SEER, la su- 
pervivencia relativa a 5 años para mujeres con cáncer de pulmón es del 18,2\% comparada con el 14,5\% en hombres. La razón del mejor pronóstico se desconoce.

Estudios poblacionales múltiples han identificado una respuesta superior al tratamiento del cáncer de pulmón en las mujeres en comparación con los hombres, independientemente del estadio, edad, modalidad terapéutica, o la histología. El proyecto Europeo Lungscape, ha recogido datos clínicos de pacientes con carcinoma de pulmón tratados quirúrgicamente con estadio IB-IIIA en 20 países europeos (36,2\% mujeres) y encontró un beneficio de supervivencia estadísticamente significativo en el sexo femenino. En relación con la enfermedad avanzada, los estudios muestran un beneficio para el sexo femenino, de forma consistente. Sin embargo, las mujeres experimentan una mayor toxicidad gastrointestinal y neuropatía. Se postula que la disminución de la capacidad de reparación del ADN en las mujeres podría ser responsable de la mayor tasa de respuesta y la toxicidad con agentes de platino. Estos estudios están limitados por la incapacidad de controlar a todos los factores como las comorbilidades y la historia de tabaquismo. En un análisis retrospectivo que agrupaba cuatro ensayos clínicos españoles y que incluía 1,191 pacientes con cáncer de pulmón avanzado (14,9\% mujeres), las mujeres tenían una mayor supervivencia que los hombres en el análisis multivariable (12.9 meses vs 9.3 meses; $p<0.001$ ) (Isla et al., 2007). La mejora de la supervivencia de las mujeres con cáncer de pulmón podría explicarse por las diferencias en la histología, historia de tabaquismo, y diferente patrón de mutación conductora entre las mujeres.

\section{CONCLUSIONES}

El cáncer de pulmón es la principal causa de muerte por cáncer y el tabaquismo es el principal factor asociado con el riesgo de desarrollar un cáncer de pulmón. Comparado con los no fumadores, los fumadores tienen 20 veces más riesgo de desarrollar un cáncer de pulmón. El cáncer de pulmón en mujeres está aumentado debido a que hay en la actualidad más mujeres fumadoras. Globalmente el porcentaje de hombres fumadores está disminuyendo lentamente mientras que el porcentaje de mujeres está aumentando. En algunas partes del mundo occidental el cáncer de pulmón es ya la primera causa de muerte por cáncer en mujeres por delante del cáncer de mama. Es por ello extremadamente importante que la población conozca esta realidad y que modifique el estilo de vida, eliminado el tabaquismo, para poder detener esta epidemia que representa el cáncer de pulmón.

\section{BIBLIOGRAFÍA}

Dogan, S., Shen, R., Ang, D. C., Johnson, M. L., D’Angelo, S. P., Paik, P. K., et al. (2012). Molecular epidemiology of EGFR and KRAS mutations in 3,026 lung adenocarcinomas: higher susceptibility of women to smoking-related KRAS-mutant cancers. Clinical Cancer Research, 18, 22, pp. 6169-6177. DOI: 1078-0432. CCR-11-3265 [pii]10.1158/1078-0432. CCR-11-3265.

Ferlay, J., Steliarova-Foucher, E., LortetTieulent, J., Rosso, S., Coebergh, J. W., Comber, H., et al. (2013). Cancer incidence and mortality patterns in Europe: estimates for 40 countries in 2012. European Journal of Cancer, 49, 6, pp. 1374-1403. DOI: S0959-8049(13)000075 [pii]10.1016/j.ejca.2012.12.027.

Fernández, E., Schiaffino, A., Borras, J. M., Shafey, O., Villalbi, J. R. y La Vecchia, C. (2003). Prevalence of cigarette smoking by birth cohort among males and females in Spain, 1910-1990. European Journal of Cancer Prevention, 12, 1, pp. 57-62. DOI: 10.1097/01. cej.0000043743.13672.a7.
Freedman, N. D., Leitzmann, M. F., Hollenbeck, A. R., Schatzkin, A. y Abnet, C. C. (2008). Cigarette smoking and subsequent risk of lung cancer in men and women: analysis of a prospective cohort study. Lancet Oncology, 9, 7, pp. 649-656. DOI: S1470-2045(08)70154-2 [pii]10.1016/S1470-2045(08)70154-2.

Fu, J. B., Kau, T. Y., Severson, R. K. y Kalemkerian, G. P. (2005). Lung cancer in women: analysis of the national Surveillance, Epidemiology, and End Results database. Chest, 127, 3, pp. 768-777. DOI: 127/3/768 [pii]10.1378/ chest.127.3.768.

Henschke, C. I., Yip, R. y Miettinen, O. S. (2006). Women's susceptibility to tobacco carcinogens and survival after diagnosis of lung cancer. Journal of the American Association, 296, 2, pp. 180-184. DOI: 296/2/180 [pii]10.1001/ jama.296.2.180.

Hsu, L. H., Chu, N. M., Liu, C. C., Tsai, S. Y., You, D. L., Ko, J. S., et al. (2009). Sexassociated differences in non-small cell lung cancer in the new era: is gender an independent prognostic factor? Lung Cancer, 66, 2, pp. 262-267. DOI: S01695002(09)00062-2 [pii]10.1016/j.lungcan.2009.01.020.

Isla, D., Felip, E., Garrido, P., Vinolas, N., Garcia-Campelo, R., Lianes, P., et al. (2007). Sex differences in non-small cell lung cancer (NSCLC) patients (p) participating in Spanish Lung Cancer Group (SLCG) trials. ASCO Meeting Abstracts, 25, 18_suppl, pp. 7679.

Isla, D., Provencio, M., Majem, M., Felip, E., Vinolas, N., Campelo, R. G., et al. (2012). Never-smoking women with lung cancer from the Spanish WORLD07 database. ASCO Meeting Abstracts, 30, 15_suppl, pp. 1531.

Janjigian, Y. Y., McDonnell, K., Kris, M. G., Shen, R., Sima, C. S., Bach, P. B., et al. (2010). Pack-years of cigarette smoking as a prognostic factor in patients with stage IIIB/IV nonsmall cell lung cancer. Cancer, 116, 3, pp. 670-675. DOI: 10.1002/cncr.24813. 
Khuder, S. A. (2001). Effect of cigarette smoking on major histological types of lung cancer: a meta-analysis. Lung Cancer, 31, 2-3, pp. 139-148. DOI: S01695002(00)00181-1 [pii].

Li, Q., Dresler, C., Heck, J. E., Allwright, S., Haglund, M., Sanchez, S., et al. (2010). Knowledge and beliefs about smoking and cancer among women in five European countries. Cancer Epidemiology, Biomarkers and Prevention, 19, 11, pp. 2811-2820. DOI: 1055-9965.EPI-10-0432 [pii]10.1158/1055-9965.EPI-10-0432.

Lortet-Tieulent, J., Soerjomataram, I., Ferlay, J., Rutherford, M., Weiderpass, E. y Bray, F. (2014). International trends in lung cancer incidence by histological subtype: adenocarcinoma stabilizing in men but still increasing in women. Lung Cancer, 84, 1, pp. 13-22. DOI: S01695002(14)00044-0 [pii]10.1016/j.lungcan.2014.01.009.

Majem, M., Artal, A., De Castro, J., Felip, E., Garrido Lopez, P., Isla, D., et al. (2012). Population survey to assess the knowledge of smoking habit and its consequences on women (w) in Spain. ASCO Meeting Abstracts, 30, 15_suppl, pp. e12000.

Malvezzi, M., Bertuccio, P., Levi, F., La Vecchia, C. y Negri, E. (2014). European cancer mortality predictions for the year 2014. Annals of Oncology, 25, 8, pp. 1650-1656. DOI: mdu138 [pii]10.1093/annonc/mdu138.

Marchetti, A., Felicioni, L., Malatesta, S., Grazia Sciarrotta, M., Guetti, L., Chella, A., et al. (2011). Clinical features and outcome of patients with non-small-cell lung cancer harboring BRAF mutations. Journal of Clinical Oncology, 29, 26, pp. 3574-3579. DOI: JCO.2011.35.9638 [pii]10.1200/JCO.2011.35.9638.

Martínez-Sánchez, J. M., Fernández, E., Fu, M., Gallus, S., Martínez, C., Sureda, X., et al. (2010). Smoking behaviour, involuntary smoking, attitudes towards smokefree legislations, and tobacco control activities in the European Union. PLOS One, 5, 11, pp. e13881. DOI: 10.1371/ journal.pone.0013881.

Mazieres, J., Peters, S., Lepage, B., Cortot, A. B., Barlesi, F., Beau-Faller, M., et al. (2013a). Lung cancer that harbors an HER2 mutation: epidemiologic characteristics and therapeutic perspectives. Journal of Clinical Oncology, 31, 16, pp. 1997-2003. DOI: JCO.2012.45.6095 [pii]10.1200/JCO.2012.45.6095.

Mazieres, J., Rouquette, I., Lepage, B., Milia, J., Brouchet, L., Guibert, N., et al. (2013b). Specificities of lung adenocarcinoma in women who have never smoked. Journal of Thoracic Oncology, 8, 7, pp. 923-929. DOI: 10.1097/JTO.0b013e3182904dfb.

Micheli, A., Ciampichini, R., Oberaigner, W., Ciccolallo, L., de Vries, E., Izarzugaza, I., et al. (2009). The advantage of women in cancer survival: an analysis of EUROCARE-4 data. European Journal of Cancer, 45, 6, pp. 1017-1027. DOI: S0959-8049(08)00913-1 [pii]10.1016/j.ejca.2008.11.008.

Nakamura, H., Ando, K., Shinmyo, T., Morita, K., Mochizuki, A., Kurimoto, N., et al. (2011). Female gender is an independent prognostic factor in non-small-cell lung cancer: a meta-analysis. Annals $f$ Thoracic and Cardiovascular Surgery, 17, 5, pp. 469-480. DOI: JST.JSTAGE/atcs/oa.10.01637 [pii].

Ng, M., Freeman, M. K., Fleming, T. D., Robinson, M., Dwyer-Lindgren, L., Thomson, B., et al. (2014). Smoking prevalence and cigarette consumption in 187 countries, 1980-2012. Journal of the American Association, 311, 2, pp. 183-192. DOI: 1812960 [pii]10.1001/ jama.2013.284692.

Paik, P. K., Johnson, M. L., D’Angelo, S. P., Sima, C. S., Ang, D., Dogan, S., et al. (2012). Driver mutations determine survival in smokers and never-smokers with stage IIIB/IV lung adenocarcinomas. Cancer, 118, 23, pp. 5840-5847. DOI: $10.1002 /$ cncr.27637.

Papadopoulous, A., Guida, F. y Leffondré, K. (2014). Heavy smoking and lung cancer: are women at high risk? Results of ICARE study. British Journal of Cancer, 110, pp. 1358-1391.

Radzikowska, E., Glaz, P. y Roszkowski, K. (2002). Lung cancer in women: age, smoking, histology, performance status, stage, initial treatment and survival. Population-based study of 20561 cases. Annals of Oncology, 13, 7, pp. 1087-1093.

Ries, L., Young, J., Keel, G., Eisner, M., Lin, Y. y Horner, M. e. (2007). SEER survival monograph: Cancer survival among adults: US SEER program, 1988-2001: patient and tumor characteristics. $\mathrm{NIH}$ publications no 07-6215. Bethesda, MD: National Cancer Institute [en línea]. [Fecha de consulta: julio 2013]. Disponible en: http://seer.cancer.gov/publications/ survival/.

Rouquette, I., Lauwers-Cances, V., Allera, C., Brouchet, L., Milia, J., Nicaise, Y., et al. (2012). Characteristics of lung cancer in women: importance of hormonal and growth factors. Lung Cancer, 76, 3, pp. 280-285. DOI: S0169-5002(11)00597-6 [pii]10.1016/j.lungcan.2011.11.023.
Sagerup, C. M., Smastuen, M. y Johannesen, T. B. (2001). Sex-specific trends in lung cancer incidence and survival: a population study of 40.118 cases. Thorax, 66, pp. 301-307.

Salmeron, D., Chirlaque, M. D., Isabel Izarzugaza, M., Sanchez, M. J., MarcosGragera, R., Ardanaz, E., et al. (2012). Lung cancer prognosis in Spain: the role of histology, age and sex. Respiratory Medicine, 106, 9, pp. 1301-1308. DOI: S0954-6111(12)00203-X [pii]10.1016/j. rmed.2012.06.006.

Sánchez, M. J., Payer, T., De Angelis, R., Larranaga, N., Capocaccia, R. y Martínez, C. (2010). Cancer incidence and mortality in Spain: estimates and projections for the period 1981-2012. Annals of Oncology, 21, Suppl 3, pp. iii30-36. DOI: mdq090 [pii]10.1093/annonc/mdq090.

Steliarova-Foucher, E., O'Callaghan, M. y Ferlay, J. (2012). European Cancer Observatory: cancer incidence, mortality, prevalence and survival in Europe. Version 1.0. European Network of Cancer Registries, International Agency for Research on Cancer [en línea]. [Fecha de consulta: febrero 2913]. Disponible en: http://eco.iarc.fr.

Torre, L. A., Siegel, R. L., Ward, E. M. y Jemal, A. (2014). International variation in lung cancer mortality rates and trends among women. Cancer Epidemiology, Biomarkers and Prevention, 23, 6, pp. 1025-1036. DOI: 1055-9965.EPI-13-1220 [pii]10.1158/1055-9965.EPI-13-1220.

Wakelee, H. A., Dahlberg, S. E., Brahmer, J. R., Schiller, J. H., Perry, M. C., Langer, C. J., et al. (2012). Differential effect of age on survival in advanced NSCLC in women versus men: analysis of recent Eastern Cooperative Oncology Group (ECOG) studies, with and without bevacizumab. Lung Cancer, 76, 3, pp. 410-415. DOI: S0169-5002(11)00668-4 [pii]10.1016/j.lungcan.2011.12.006.

Wheatley-Price, P., Blackhall, F., Lee, S. M., Ma, C., Ashcroft, L., Jitlal, M., et al. (2010). The influence of sex and histology on outcomes in non-small-cell lung cancer: a pooled analysis of five randomized trials. Annals of Oncology, 21, 10, pp. 2023-2028. DOI: mdq067 [pii]10.1093/annonc/mdq067.

Zang, E. A. y Wynder, E. L. (1996). Differences in lung cancer risk between men and women: examination of the evidence. Journal of the National Cancer Institute, 88, 3-4, pp. 183-192. 